Received: 18.03 .2021

Revised: 20.04 .2021

Accepted: 23.04 .2021

DOI: $10.17804 / 2410-9908.2020 .2 .064-072$

\title{
SELECTION OF COMPLEX EQUIVALENT STRESS FOR TWO DIFFERENT VARIANTS OF THE PLANE STRESS STATE
}

\author{
V. V. Nazarov \\ Institute of Mechanics, Lomonosov Moscow State University \\ 1 Michurinsky Ave., Moscow, 119192, Russian Federation \\ iDhttps://orcid.org/0000-0002-9234-3646 inmec130@mail.ru \\ Corresponding author. E-mail: inmec130@mail.ru \\ Address for correspondence: 1 Michurinsky Ave., Moscow, 119192, Russian Federation
}

To describe the creep rupture process under complex stress, various equivalent stresses are considered. From them, the equivalent stress at which the total error of the difference between the experimental and theoretical values takes the smallest value among the considered equivalent stresses is selected. In this paper, three basic equivalent stresses are considered, as well as two complex equivalent stresses, which are a linear combination of the basic ones with one material parameter. The analysis of the total errors in the considered experimental data shows that, with the simultaneous effect of internal pressure and the axial force on the wall of tubular specimens (or biaxial tension of a plane element), a complex equivalent stress should be used in the form of a combination of the maximum normal stress and the Mises stress. For simultaneous torsion and tension of tubular specimens (or simultaneous tension and compression of a plane element), a complex equivalent stress should be used in the form of a combination of the maximum normal stress and the doubled maximum tangential stress.

Keywords: creep rupture, time at rupture, plane stress state, equivalent stress.

\section{Acknowledgment}

The study was partially financially supported by the Russian Foundation for Basic Research, grant 20-08-00387.

\section{References}

1. Lokoshchenko A.M., Nazarov V.V. Kinetic approach of investigation of creep-rupture for metals under biaxial tension. Aviatsionno-Kosmicheskaya Tekhnika i Tekhnologiya, 2005, no. 10 (26), pp. 73-79. (In Russian). Available at: http://nbuv.gov.ua/UJRN/aktit_2005_10_15

2. Lokoshchenko A.M., Nazarov V.V. Choice of Long-Term Strength Criteria for Metals in Combined Stress State. Aviatsionno-Kosmicheskaya Tekhnika i Tekhnologiya, 2004, no. 7 (15), pp. 124-128. (In Russian). Available at: http://nbuv.gov.ua/UJRN/aktit_2004_7_27

3. Lokoshchenko A.M. Long-term strength of metals in complex stress state (a survey). Mechanics of Solids, 2012, vol. 47, pp. 357-372. DOI: 10.3103/S0025654412030090.

4. Himeno T., Chuman Y., Tokiyoshi T., Fukahori T., Igari T. Creep rupture behaviour of circumferentially welded mod. $9 \mathrm{Cr}-1 \mathrm{Mo}$ steel pipe subject to internal pressure and axial load. Materials at High Temperatures, 2016, vol. 33, iss. 6, pp. 636-643. DOI: 10.1080/09603409.2016.1226703.

5. Kobayashi H., Ohki R., Itoh T., Sakane M. Multiaxial creep damage and lifetime evaluation under biaxial and triaxial stresses for type 304 stainless steel. Engineering Fracture Mechanics, 2017, vol. 174, pp. 30-43. DOI: 10.1016/j.engfracmech.2017.01.001. 
6. Dyson B.F., Mclean D. Creep of Nimonic 80A in torsion and tension. Metal Science, 1977, vol. 11, iss. 2, pp. 37-45. DOI: 10.1179/msc.1977.11.2.37.

7. Cane B.J. Creep damage accumulation and fracture under multiaxial stresses. In: Proceedings of The 5th International Conference on Fracture Mechanics «Advances in Fracture Research», Cannes, France, 29 March-2 April 1981, Oxford, 1981, vol. 3, pp. 1285-1293.

8. Nazarov V.V. Determination of creep properties under tension and torsion of copper tubular specimens. Inorganic Materials, 2014, vol. 50, pp. 1514-1515. DOI: 10.1134/S0020168514150138.

9. Kowalewski Z.L. Biaxial creep study of copper on the basis of isochronous creep surfaces. Archives of Mechanics, 1996, vol. 48, no 1, pp. 89-109. Available at: https://am.ippt.pan.pl/am/article/view/v48p89

10. Stanzl-Tschegg S., Argon A.S., Tschegg E.K. Diffusive intergranular cavity growth in creep in tension and torsion. Acta Metallurgica, 1983, vol. 31, iss. 6, pp. 833-843. DOI: 10.1016/00016160(83)90111-6.

11. Nazarov V.V. Criterion of creep rupture for tubular specimens under tension and torsion. Industrial Laboratory. Diagnostics of Materials, 2014, vol. 80, no. 12, pp. 57-59. (In Russian).

12. Lebedev A.A. The theory of equivalent stresses as a problem of mechanics of materials. Strength of Materials, 1996, vol. 28, no. 2, pp. 94-108. DOI: 10.1007/BF02215833.

13. Nazarov V.V. Analysis of two creep rupture models. Diagnostics, Resource and Mechanics of materials and structures, 2019, iss. 5, pp. 73-80. DOI: 10.17804/2410-9908.2019.5.073-080. Available at: https://dream-journal.org/DREAM_Issue_5_2019_Nazarov_V.V._073_080.pdf

14. Nazarov V.V. Approximation of secondary creep for tubular specimens under tension and torsion. Industrial Laboratory. Diagnostics of Materials, 2015, vol. 81, no 7, pp. 60-61. (In Russian).

15. Norton F.N. Creep of Steel at High Temperatures, New York, Mc. Graw-Hill Book Company, 1929, $67 \mathrm{p}$.

16. Bailey R.W. Creep of steel under simple and compound stresses and the use of high initial temperature in steam power plant. In: Transactions of World Power Conference, Oct-Nov 1929, Tokyo, vol. 3.

17. Lasdon L.S., Fox R.L., Ratner M.W. Nonlinear optimization using the generalized reduced gradient method. Operations Research, 1974, vol. 8, No. V3, pp. 73-103. Available at: http://www.numdam.org/item/RO_1974_8_3_73_0/

18. Available at: https://www.solver.com/excel-solver-algorithms-and-methods-used 
Подана в журнал: 18.03 .2021

УДК 539.376

DOI: $10.17804 / 2410-9908.2021 .2 .064-072$

\title{
ВЫБОР СЛОЖНОГО ЭКВИВАЛЕНТНОГО НАПРЯЖЕНИЯ ДЛЯ ДВУХ РАЗЛИЧНЫХ ВАРИАНТОВ ПЛОСКОГО НАПРЯЖЕННОГО СОСТОЯНИЯ
}

\author{
В. В. Назаров
}

\author{
Научно-исследовательский институт механики \\ Федерального государственного бюджетного образовательного учреждения высшего образования \\ «Московский государственный университет имени М.В. Ломоносова» \\ Мичуринский проспект, 1, Москва, Российская Федераџия \\ (iD https://orcid.org/0000-0002-9234-3646 inmec130@mail.ru \\ Ответственный автор. Электронная почта: inmec130@ mail.ru \\ Адрес для переписки: Мичуринский проспект, 1, Москва, 119192, Российская Федерация
}

Для описания процесса длительной прочности при сложном напряженном состоянии рассматривают различные эквивалентные напряжения. Из них выбирают такое эквивалентное напряжение, при котором суммарная погрешность разности экспериментального и теоретического значений принимает наименьшее значение среди всех рассмотренных эквивалентных напряжений. В работе рассмотрены три базовых эквивалентных напряжения, а также два сложных эквивалентных напряжения, представляющих собой линейную комбинацию базовых с одним материальным параметром. Анализ суммарных погрешностей в рассмотренных экспериментальных данных показал, что при одновременном воздействии внутреннего давления и осевой силы на стенку трубчатых образцов (или двухосном растяжении плоского элемента) следует использовать сложное эквивалентное напряжение в виде комбинации максимального нормального напряжения и напряжения Мизеса. При одновременном кручении и растяжении трубчатых образцов (или одновременном растяжении и сжатии плоского элемента) следует использовать сложное эквивалентное напряжение в виде комбинации максимального нормального напряжения и удвоенного максимального касательного напряжения.

Ключевые слова: длительная прочность, время в момент разрушения, плоское напряженное состояние, эквивалентное напряжение.

\section{1. Введение}

В работе рассмотрена длительная прочность изотропных металлических материалов (металлы, стали, никелевые сплавы), в основе строения которых присутствует кристаллическая решетка. Для анизотропных металлических материалов в элементах конструкций дополнительно рассмотрен коэффициент прочностной анизотропии, что никак не влияет на полученный результат. При исследовании явления длительной прочности отчет времени начинают с момента приложения стационарных сил. Под действием этих сил в материале накапливаются рассеянные повреждения, которые приводят к образованию магистральных трещин и разрушению в определенный момент времени, поэтому в качестве характеристики явления длительной прочности рассматривают время в момент разрушения.

Для описания длительной прочности при сложном напряженном состоянии используют либо кинетический [1], либо критериальный подход [2]. В обзоре [3] перечислены экспериментальные и теоретические результаты, полученные в условиях сложного напряженного состояния. Каждый из двух подходов применяется в зависимости от типа 
сложного напряженного состояния, которое определяется знаками главных напряжений $\sigma_{1} \geq \sigma_{2} \geq \sigma_{3}$. Кинетический подход [1] используют при описании длительной прочности либо при двухосном растяжении $\left(\sigma_{1} \geq \sigma_{2}>0, \sigma_{3}=0\right)$ элементарного плоского элемента (испытания на совместное внутреннее давление и растяжение трубчатых образцов [4] или двухосное растяжение плоских образцов [5]), либо при трехосном растяжении ( $\sigma_{1} \geq \sigma_{2} \geq \sigma_{3}>0$ ) элементарного объемного элемента [5] (испытания на одновременное трехосное растяжение кубических образцов). Суть кинетического подхода [1] заключается в том, что повреждения накапливаются исключительно на площадках, перпендикулярных главным растягивающим напряжениям, на которых скорости накопления компонент вектора поврежденности пропорциональны соответствующим главным напряжениям, что означает $\frac{d \omega_{1}}{d t}=f\left(\sigma_{1}\right), \frac{d \omega_{2}}{d t}=f\left(\sigma_{2}\right)$ и $\frac{d \omega_{3}}{d t}=f\left(\sigma_{3}\right)$, где $t-$ время воздействия стационарных сил. В качестве характеристики поврежденного состояния материала рассматривается абсолютная величина вектора поврежденности $\omega=\sqrt{\omega_{1}^{2}+\omega_{2}^{2}+\omega_{3}^{2}}$ с двумя условиями по времени $\omega_{t=0}=0$ и $\left.\omega\right|_{t=t_{\text {rupt }}}=1$, где $t_{\text {rupt }}-$ время в момент разрушения. В частном случае, когда одно из трех главных напряжений равно нулю (в этом частном случае многоосного растяжения одна из компонент вектора поврежденности $\left.\omega_{3}=0\right)$, абсолютная величина вектора поврежденности принимает вид $\omega=\sqrt{\omega_{1}^{2}+\omega_{2}^{2}}$. Время в момент разрушения $t_{\text {rupt }}$ определяется из выражения для абсолютной величины $\omega$ вектора поврежденности при интегрировании от начального состояния $\left.\omega\right|_{t=0}=0$ до состояния в момент разрушения $\left.\omega\right|_{t=t_{\text {rupt }}}=1$.

Обратим внимание, что кинетический подход [1] не позволяет описать длительную прочность при одновременном растяжении и сжатии $\left(\sigma_{1}>0 ; \sigma_{2}=0 ; \sigma_{3}<0\right)$ элементарного плоского элемента по двум взаимно ортогональным направлениям [6-10] (испытания на одновременное кручение и растяжение трубчатых образцов). Для главных напряжений следует использовать критериальный подход [2], в котором принято, что $t_{\text {rupt }}=g\left(\sigma_{\text {eq }}\right)$, где $\sigma_{\text {eq }}-$ эквивалентное напряжение (одна из скалярных характеристик тензора напряжений). Скалярные характеристики выбираются таким образом, чтобы они принимали одинаковое значение при одноосном растяжении (исключением является модель [11]). Как правило, при анализе суммарных погрешностей $\Delta$ (формула приведена ниже) рассматривают три основных эквивалентных напряжения (максимальное нормальное напряжение $\sigma_{\max }$, напряжение Мизеса $\sigma_{\text {mises }}$ и удвоенное максимальное касательное напряжение $2 \tau_{\max }$ ). Дополнительно к трем основным эквивалентным напряжениям, могут рассматриваться их возможные линейные комбинации с одним материальным параметром, где величина этого параметра определяет вклад одного из двух используемых базовых эквивалентных напряжений на время в момент разрушения. 


\section{2. Два сложных эквивалентных напряжения}

Рассмотрим два сложных эквивалентных напряжения $\sigma_{\text {eq }}^{1}$ [12] и $\sigma_{\text {eq }}^{2}$ [13] с параметрами $\beta_{1}$ и $\beta_{2}$ в виде линейных комбинаций из разных базовых эквивалентных напряжений:

$$
\begin{gathered}
\sigma_{\mathrm{eq}}^{1}=\left[1-\beta_{1}\right] \sigma_{\text {mises }}+\beta_{1} \sigma_{\max }, \quad 0 \leq \beta_{1} \leq 1 ; \\
\sigma_{\mathrm{eq}}^{2}=\left[1-\beta_{2}\right]\left[2 \tau_{\max }\right]+\beta_{2} \sigma_{\max }, \quad 0 \leq \beta_{2} \leq 1 .
\end{gathered}
$$

Из анализа суммарных погрешностей постараемся определить, какое из двух рассмотренных сложных эквивалентных напряжений $\sigma_{\text {eq }}^{1}$ и $\sigma_{\text {eq }}^{2}$ следует использовать при описании длительной прочности для каждого из двух возможных типов плоского напряженного состояния. Чтобы убедиться в целесообразности рассмотренных сложных эквивалентных напряжений, для анализа потребуется вычислить суммарные погрешности основных эквивалентных напряжений.

\section{3. Методика вычисления суммарных погрешностей}

В статье [13] представлены результаты анализа минимальных суммарных погрешностей для трех основных эквивалентных напряжений $\sigma_{\max }, \sigma_{\operatorname{mises}}, 2 \tau_{\max }$ и двух сложных эквивалентных напряжений $\sigma_{\text {eq }}^{1}$ и $\sigma_{\text {eq }}^{2}$. Установлено, что для аппроксимации используемых для анализа экспериментальных данных при $\sigma_{1}>0, \sigma_{2}=0$ и $\sigma_{3}<0$, полученных на трубчатых образцах под действием кручения и растяжения, следует использовать эквивалентное напряжение (2). Данная работа является продолжением [13], где дополнительно будут рассмотрены экспериментальные данные при $\sigma_{1}>0, \sigma_{2}=0$ и $\sigma_{3}<0$, полученные на трубчатых образцах под действием внутреннего давления и растяжения и плоских образцах под действием двух взаимно ортогональных сил.

При двухосном растяжении элементарного плоского элемента

$$
\sigma_{\text {max }}=\sigma_{1}, \quad \sigma_{\text {mises }}=\sqrt{\sigma_{1}^{2}-\sigma_{1} \sigma_{2}+\sigma_{2}^{2}}, \quad 2 \tau_{\max }=\sigma_{1} \text {. }
$$

Можно заметить, что $\sigma_{\max }=2 \tau_{\max }=\sigma_{1}$, что означает $\sigma_{\text {eq }}^{2}=\sigma_{1}$.

При одновременном растяжении и сжатии элементарного плоского элемента

$$
\sigma_{\max }=\sigma_{1}, \quad \sigma_{\text {mises }}=\sqrt{\sigma_{1}^{2}-\sigma_{1} \sigma_{3}+\sigma_{3}^{2}}, \quad 2 \tau_{\max }=\sigma_{1}-\sigma_{3},
$$

где главные напряжения [14] зависят от нормального напряжения $\sigma$ и касательного напряжения $\tau$ следующим образом:

$$
\sigma_{1}=\frac{\sigma}{2}+\sqrt{\left[\frac{\sigma}{2}\right]^{2}+\tau^{2}}, \quad \sigma_{2}=0, \quad \sigma_{3}=\frac{\sigma}{2}-\sqrt{\left[\frac{\sigma}{2}\right]^{2}+\tau^{2}} .
$$

Для связи времени в момент разрушения от эквивалентного напряжения будем использовать степенную зависимость с двумя материальными параметрами $[15,16]$ : 


$$
\frac{t_{\mathrm{rupt}}^{\mathrm{app}}}{B}=\left[\frac{\sigma_{\mathrm{eq}}}{\sigma_{\mathrm{dim}}}\right]^{-m}, \quad B>0, \quad m>1,
$$

где $t_{\text {rupt }}^{\text {app }}$ - время в момент разрушения из аппроксимации; $\sigma_{\text {eq }}-$ одно из рассматриваемых эквивалентных напряжений $\sigma_{\max } ; \sigma_{\operatorname{mises}} ; 2 \tau_{\max } ; \sigma_{\mathrm{eq}}^{1}$ и $\sigma_{\mathrm{eq}}^{2} ; B ; m$ - материальные параметры; $\sigma_{\text {dim }}=1 \mathrm{MPa}$ - обезразмеривающее напряжение.

Неизвестные параметры $B, m$ и $\beta_{1}$ (или $\beta_{2}$ ) вычисляются из условия минимальной суммарной погрешности разности экспериментальных $t_{\text {rupt }}^{\exp }$ и их аппроксимирующих $t_{\text {rupt }}^{\text {app }}$ значений времени в момент разрушения:

$$
\Delta=\min \left(\sum_{1}^{N}\left|\lg \frac{t_{\text {rupt }}^{\text {app }}}{\mathrm{t}_{\text {rupt }}^{\exp }}\right|\right),
$$

где $N$ - количество экспериментов в данной серии; $t_{\text {rupt }}^{\exp }-$ время в момент разрушения из эксперимента. Выражение (7) является аналогом метода наименьших квадратов, где в качестве расстояния между двумя значениями используется модуль разности логарифмов $\lg \left(t_{\text {rupt }}^{\text {app }}\right)-\lg \left(t_{\text {rupt }}^{\text {exp }}\right)$, который равен логарифму отношения $\lg \left(t_{\text {rupt }}^{\text {app }} / t_{\text {rupt }}^{\text {exp }}\right)$. Минимизация целевого условия (7) реализуется через алгоритм [17] нелинейной оптимизации Обобщенного метода сопряженных градиентов (GRG2) с помощью меню надстройки в Microsoft Excel [18]. Для неизвестных параметров $B, m$ и $\beta_{1}$ (или $\beta_{2}$ ) начальное приближение их значений используется из равенства $t_{\text {rupt }}^{\text {app }}=t_{\text {rupt }}^{\text {exp }}$.

\section{4. Экспериментальные данные для анализа суммарных погрешностей}

В работе при анализе суммарных погрешностей рассмотрены экспериментальные данные, которые получены для различных изотропных металлических материалов разных марок стали, никелевого сплава и меди после термической обработки (табл. 1). Влияние прочностной анизотропии на время в момент разрушения прослеживается в экспериментальных данных, полученных при одновременном кручении и растяжении трубчатых образцов [9-10]. При аппроксимации экспериментальных данных [9-10] наблюдается эффект нескольких аппроксимаций [11]. Этот эффект заключается в том, что аппроксимация зависит от типа напряженного состояния (чистое растяжение, чистое кручение, одновременное кручение и растяжение). Для того чтобы экспериментальные значения подчинялись одной единственной эмпирической закономерности, перед началом механических испытаний образцы подвергают термической обработке, которая убирает остаточные напряжения и преобразует микроструктуру до равноосной с хаотической ориентацией границ зерен. 
Таблица 1 - Сводная таблица публикаций экспериментальных данных при двух разных плоских напряженных состояниях

\begin{tabular}{|c|c|c|c|c|}
\hline $\begin{array}{c}\text { Номер } \\
\text { эксперимента }\end{array}$ & $\begin{array}{c}\text { Ссылка } \\
\text { на источник }\end{array}$ & Материал & $\begin{array}{c}\text { Температура, } \\
{ }^{\circ} \mathrm{C}\end{array}$ & $\begin{array}{c}\text { Тип плоского } \\
\text { напряженного } \\
\text { состояния } \\
\end{array}$ \\
\hline 1 & [4] & $\begin{array}{c}\text { Сталь } \\
\text { 9Cr1MoVNb }\end{array}$ & 650 & $\begin{array}{c}\text { Одновременное } \\
\text { двухосное }\end{array}$ \\
\hline 2 & [5] & Сталь 304 & 650 & растяжение \\
\hline 3 & [6] & $\begin{array}{c}\text { Никелевый } \\
\text { сплав } \\
\text { Nimonic 80A }\end{array}$ & 750 & \multirow[t]{3}{*}{$\begin{array}{c}\text { Одновременное } \\
\text { растяжение } \\
\text { и сжатие }\end{array}$} \\
\hline 4 & [7] & $\begin{array}{c}\text { Сталь } \\
\text { 2.25Сr1Mo }\end{array}$ & 565 & \\
\hline 5 & [8] & $\begin{array}{c}\text { Медь после } \\
\text { отжига }\end{array}$ & 264 & \\
\hline
\end{tabular}

\section{5. Анализ минимальных суммарных погрешностей}

Ниже (табл. 2) приведены суммарные погрешности $\Delta$ (наименьшие погрешности подчеркнуты) и значения параметров $\beta_{1}$ и $\beta_{2}$ для сложных эквивалентных напряжений. В экспериментах 2 и 5 сложные эквивалентные напряжения оказались частным случаем базовых эквивалентных напряжений, где $\sigma_{\text {eq }}^{1}=\sigma_{\text {mises }}$ в эксперименте 2 и $\sigma_{\text {eq }}^{2}=\tau_{\max }$ в эксперименте 5.

Таблица 2 - Минимальные суммарные погрешностии параметры в сложных эквивалентных напряжениях ${ }^{*}$

\begin{tabular}{|c|c|c|c|c|c|c|c|}
\hline \multirow{2}{*}{$\begin{array}{c}\text { Номер } \\
\text { эксперимента }\end{array}$} & \multicolumn{4}{|c|}{ Суммарные погрешности } & \multicolumn{3}{c|}{ Параметры } \\
\cline { 2 - 8 } & $\sigma_{\max }$ & $\sigma_{\text {mises }}$ & $2 \tau_{\max }$ & $\sigma_{\text {eq }}^{1}$ & $\sigma_{\text {eq }}^{2}$ & $\beta_{1}$ & $\beta_{2}$ \\
\hline 1 & 0,9 & 0,8 & 0,9 & $\underline{0,6}$ & 0,9 & 0,3 & - \\
2 & 1,5 & $\underline{1,3}$ & 1,5 & $\underline{1,3}$ & 1,5 & 0,0 & - \\
\hline 3 & 4,3 & 4,3 & 6,6 & $\underline{1,6}$ & $\underline{1,7}$ & 0,5 & 0,6 \\
5 & 1,6 & 2,5 & 3,2 & $\underline{0,7}$ & $\underline{0,7}$ & 0,7 & 0,8 \\
\hline 5 & 0,6 & 0,5 & $\underline{0,3}$ & 0,5 & $\underline{0,3}$ & 0,0 & 0,0 \\
\hline
\end{tabular}

* подчеркнуты наиболее благоприятные значения суммарных погрешностей. 

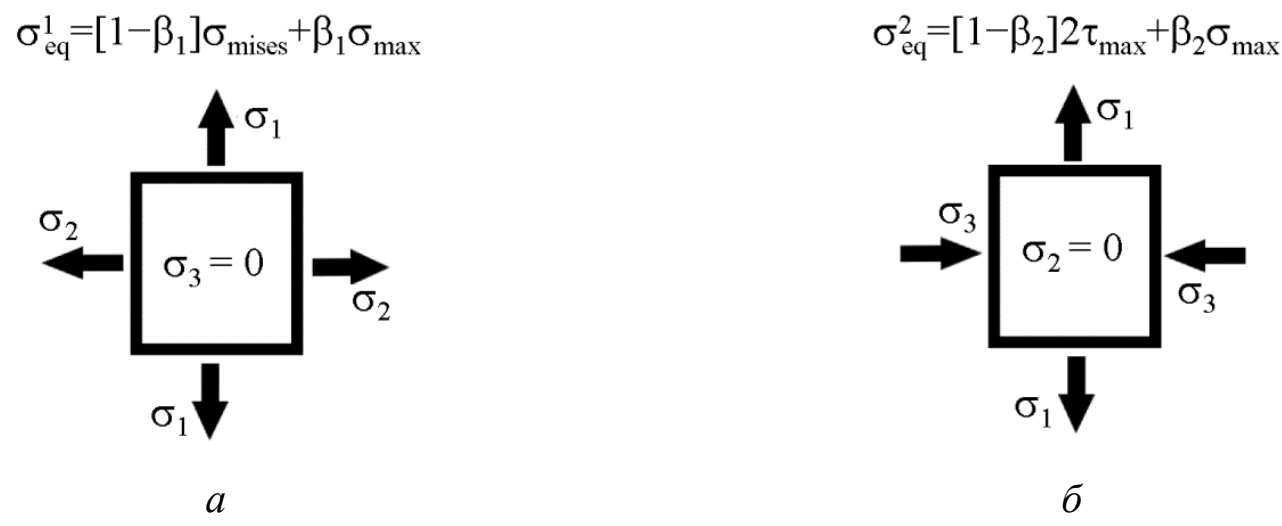

Выбор сложного эквивалентного напряжения для описания длительной прочности в зависимости от типа плоского напряженного состояния: $a$ - двухосное напряжение;

$\sigma$ - одновременное напряжение (растяжение-сжатие)

В экспериментах 3 и 4 суммарные погрешности обоих сложных эквивалентных напряжений $\sigma_{\text {eq }}^{1}$ и $\sigma_{\text {eq }}^{2}$ приняли почти одинаковые значения, при этом они заметно меньше суммарных погрешностей для базовых эквивалентных напряжений. В целом, на основе анализа всех рассмотренных экспериментальных данных, можно сделать вывод, что для описания обоих типов плоского напряженного состояния не существует общего эквивалентного напряжения. Это означает, что для каждого типа плоского напряженного состояния следует использовать свое эквивалентное напряжение (рисунок).

\section{6. Заключение}

В работе сделана попытка решить вопрос о выборе эквивалентного напряжения, позволяющего описать длительную прочность для двух разных плоских напряженных состояний, различающихся знаком одного из главных напряжений. Рассмотрены три базовых и два сложных эквивалентных напряжений с параметром. В качестве критерия отбора эквивалентного напряжения принималась минимальная суммарная погрешность разности экспериментальных и теоретических значений времени в момент разрушения. Анализ значений суммарных погрешностей показал, что для каждого типа плоского напряженного состояния следует использовать конкретное сложное эквивалентное напряжения с параметром. Для описания времени в момент разрушения в условиях двухосного растяжения следует использовать сумму максимального нормального напряжения и напряжения Мизеса. Для описания длительной прочности при одновременном растяжении и сжатии элементарного плоского элемента в двух взаимно ортогональных направлениях в качестве эквивалентного напряжения следует использовать сумму максимального нормального напряжения и удвоенного максимального касательного напряжения.

Работа посвящена выбору силового критерия прочности, эмпирически наиболее точно описывающему длительную прочность в соответствии с экспериментальными данными для двух схем плоского напряженного состояния. На основе минимизации суммарной погрешности разности экспериментальных и аппроксимирующих значений времени в момент разрушения проведено обоснование выбора сложного эквивалентного напряжения, наиболее точно описывающего длительную прочность для конкретной схемы напряженного состояния изотропного материала в соответствии с экспериментальными данными. Результаты работы имеют практическую значимость и могут быть использованы при выполнении проектных расчетов соответствующих элементов конструкций и деталей машин, работающих при статистических нагрузках в условиях плоского напряженного состояния. 


\section{Благодарность}

Работа выполнена при частичной финансовой поддержке Российского фонда фундаментальных исследований (грант 20-08-00387).

\section{Литература}

1. Локощенко А. М., Назаров В. В. Кинетический подход исследования длительной прочности металлов при двухосном растяжении // Авиационно-космическая техника и технология. - 2005. - № 10 (26). - C. 73-79. - URL: http://nbuv.gov.ua/UJRN/aktit_2005_10_15

2. Локощенко А. М., Назаров В. В. Выбор критериев длительной прочности металлов при сложном напряженном состоянии // Авиационно-космическая техника и технология. 2004. - № 7 (15). - C. 124-128. - URL: http://nbuv.gov.ua/UJRN/aktit_2004_7_27

3. Lokoshchenko A. M. Long-term strength of metals in complex stress state (a survey) // Mechanics of Solids. - 2012. - Vol. 47. - P. 357-372. - DOI: 10.3103/S0025654412030090.

4. Creep rupture behavior of circumferentially welded mod. 9Cr-1Mo steel pipe subject to internal pressure and axial load / T. Himeno, Y. Chuman, T. Tokiyoshi, T. Fukahori, T. Igari // Materials at High Temperatures. - 2016. - Vol. 33, iss. 6. - P. 636-643. - DOI: 10.1080/09603409.2016.1226703.

5. Kobayashi H., Ohki R., Itoh T., Sakane M. Multiaxial creep damage and lifetime evaluation under biaxial and triaxial stresses for type 304 stainless steel // Engineering Fracture Mechanics. 2017. - Vol. 174. - P. 30-43. - DOI: 10.1016/j.engfracmech.2017.01.001.

6. Dyson B. F., Mclean D. Creep of Nimonic 80A in torsion and tension // Metal Science. 1977. - Vol. 11, iss. 2. - P. 37-45. - DOI:10.1179/msc.1977.11.2.37.

7. Cane B. J. Creep damage accumulation and fracture under multiaxial stresses // The 5th International Conference on Fracture Mechanics «Advances in Fracture Research», Cannes, France, 29 March-2 April 1981 : proceedings. - Oxford, 1981. - Vol. 3. - P. 1285-1293.

8. Nazarov V. V. Determination of creep properties under tension and torsion of copper tubular specimens // Inorganic Materials. - 2014. - Vol. 50. - P. 1514-1515. - DOI: 10.1134/S0020168514150138.

9. Kowalewski Z. L. Biaxial creep study of copper on the basis of isochronous creep surfaces // Archives of Mechanics. - 1996. - Vol. 48, No 1. - P. 89-109. - URL: https://am.ippt.pan.pl/am/article/view/v48p89

10. Stanzl-Tschegg S., Argon A. S., Tschegg E. K. Diffusive intergranular cavity growth in creep in tension and torsion // Acta Metallurgica. - 1983. - Vol. 31, iss. 6. - P. 833-843. DOI: 10.1016/0001-6160(83)90111-6.

11. Назаров В. В. Критерий длительной прочности при растяжении и кручении трубчатых образцов // Заводская лаборатория. Диагностика материалов. - 2014. - Т. 80, № 12. - С. 57-59.

12. Lebedev A. A. The theory of equivalent stresses as a problem of mechanics of materials // Strength of Materials. - 1996. - Vol. 28, No. 2. - P. 94-108. - DOI: 10.1007/BF02215833.

13. Nazarov V. V. Analysis of two creep rupture models // Diagnostics, Resource and Mechanics of materials and structures. - 2019. - Iss. 5. - P. 73-80. - DOI: 10.17804/2410-9908.2019.5.073-080. URL: https://dream-journal.org/DREAM_Issue_5_2019_Nazarov_V.V._073_080.pdf

14. Назаров В. В. Описание установившейся ползучести при растяжении и кручении трубчатых образцов // Заводская лаборатория. Диагностика материалов. - 2015. - Т. 81, № 7. - С. 60-61.

15. Norton F. N. Creep of Steel at High Temperatures. - New York : Mc. Graw-Hill Book Company, 1929. $-67 \mathrm{p}$.

16. Bailey R. W. Creep of steel under simple and compound stresses and the use of high initial temperature in steam power plant // Transactions of World Power Conference, Tokyo, Oct-Nov 1929. - Vol. 3.

17. Lasdon L. S., Fox R. L., Ratner M. W. Nonlinear optimization using the generalized reduced gradient method // Operations Research. - 1974. - Vol. 8, No. V3. - P. 73-103. - Available at: http://www.numdam.org/item/RO_1974_8_3_73_0/

18. Available at: https://www.solver.com/excel-solver-algorithms-and-methods-used 